\title{
Design of a New-style Escort Mechanism of Vertical Internal Broaching Machine
}

\author{
Shenshun Ying \\ Ministry of Education Key Laboratory of Special Purpose \\ Equipment and Advanced Processing Technology, \\ Zhejiang University of Technology \\ Hangzhou, Zhejiang, PR China \\ yss@mail.zjut.edu.cn
}

\author{
Zhiying Yuan \\ Jinyun Gaoxin Machinery Manufacture Co., Ltd \\ Jinyun, Zhejiang, PR China
}

Co., Ltd

\author{
Minghui Feng
Jinyun Gaoxin Machinery Manufacture Co., Ltd \\ Minghui Feng
Jinyun Gaoxin Machinery Manufacture Co., Ltd \\ Jinyun, Zhejiang, PR China \\ fengminghuifmh@126.com
}

\begin{abstract}
In an effort to improve the property of escort mechanism of vertical internal broaching machine, a new-style escort mechanism is designed. Work process and defect about structure of escort mechanism in existence is firstly analyzed in depth, design concept and implementation methods of the new escort mechanism are then studied. Three-dimensional structure and work process are presented, and performance comparison between the new-style structure and the old one is conducted. Application study is launched at last. The innovative escort mechanism and slide board are movably connected and lumped together. In consequence, the escort mechanism will stop and then be hung at the column, while the tool slide continues to move down until broaching stop. The new-style escort mechanism has superiority such as low cost, high robust and good safety, and so on, which make it adaptive and useful for structural optimization of broaching machine.
\end{abstract}

Keywords-vertical internal broaching machine; escort mechanism; tool slide; column.

\section{INTRODUCTION}

Broaching is a unique process in the production of components with complex profiles, i.e. dovetail slot on compressor discs, to achieve high surface integrity and geometrical accuracy. Broaching is carried out through a sequence of roughing, semi-finishing and finishing operation in single stroke that pushes a cutting tool through the surface of the component. Aerospace and automotive manufacturers extensively employ broaching to generate tightly toleranced profiles at high surface finish specifications ${ }^{[1-4]}$. Due to the long distance of broaching machine, fishtailing will occur when broaching internal round hole, in consequence, ruin severely the broaching precision of workpiece. Consequently, a matched mechanism, namely escort mechanism of broaching tool, is necessary to guarantee the stability of broaching. As the escort mechanism of broaching tool holds important implications for precision of workpiece, it is imperative to research thoroughly

This work is partially supported by the Natural Science Foundation of Zhejiang Province (Grant No. Y1110953), Zhejiang Youth Science \& Technology Talents Cultivation Project for International Conference and Scientific Research Fund of Zhejiang University of Technology(Grant No. 20100208) to Shenshu Ying on design of innovative escort mechanism for broaching machine.

The escort mechanism of broaching tool should meet the three requirements ${ }^{[5-8]}$ : (1) synchronization. The pull chuck and escort mechanism are move synchronously, and they are relative static to broaching tool, otherwise, the broaching tool will be snapped, (2) safety. The escort mechanism is not allowed to hit the workpiece, and escorting should be broke up after a certain distance, and (3) stability. Escorting will take a long time, and should keep the broaching tool run steadily. Among them, the synchronism is able to meet the requirements so far. There are two kinds of method to guarantee safety of escorting: mechanical method, which needs complex crashavoidance mechanism; and electronic method, which exists hidden danger of electronic device failure. Consequently, the safety of escorting remains to be further improved. As to the stability, escorting distance in existence accounts for only a third of the length of broaching tool, which ruin severely the broaching precision of workpiece and have to await further enhancements.

This paper presents a new-style escort mechanism of broaching machine. Design concept and implementation methods of the new escort mechanism are introduced. Threedimensional structure and work process are presented, and performance comparison between the new-style structure and the old one is conducted. Application study is launched to validate the design results at last.

\section{ANALYSIS OF CURRENT ESCORT MECHANISM}

A. Vertical internal broaching machine

Figure 1 shows a typical mechanical structure of vertical internal broaching machine. It consists of base box, column, base table, tool slide, work table, escort mechanism, etc. base box, column and base table are rigidly connected. The column provides mounting surface for linear rails. Workpiece is fixed on the base table and work table. The tool slide moves up and 
down on linear rails. The base box is capacity of wearing debris from debris and chip removal. Escort mechanism in charge of escorting of broaching tool. Broaching is an intermittent cutting process due to the repetitive entrances/exits of the cutting teeth into/from the workpiece.
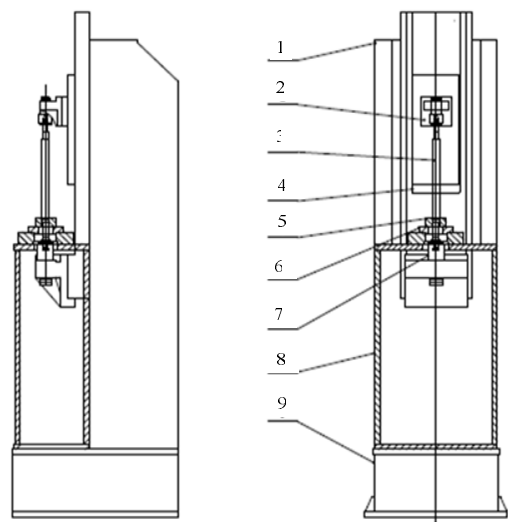

Fig.1. Mechanical structure of the vertical internal broaching machine .(1column 2- escort mechanism 3- broaching tool 4- tool slide 5- workpiece 6work table 7- pull block 8- table base 9- base box.)

\section{B. Escorting process of existing escort mechanism}

Figure 2 shows escorting process of an existing typical escort mechanism of vertical internal broaching machine. The escort mechanism is installed in the tool slide, and they moves relative to each other. The escort mechanism holds the tail of the broaching tool during the broaching operation. The escorting process includes three stages: I. broaching starts; II. escorting stops; III. broaching stops. In the first stage, the escort mechanism holds the tail of the broaching tool and moves along with the tool slide. There is no relative movement between the escort mechanism and the tool slide. The pull block keep pace with the escort mechanism until the escorting stops, namely, stages II. Now, the escort mechanism is close to the workpiece. The escort mechanism then moves up in opposite directions to prevent the collision, while the tool slide keeps moving down until the broaching stops.

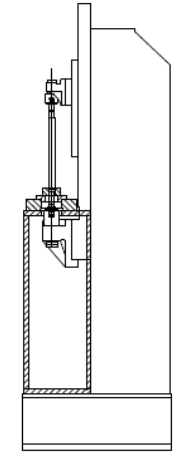

Stage I

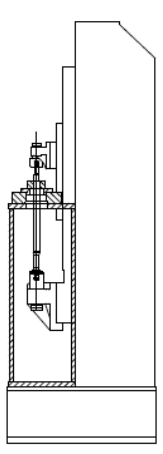

Stage II

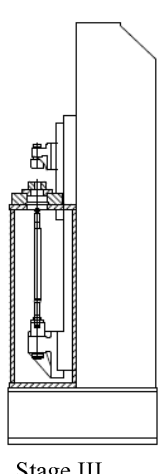

Stage III
Fig.2. Escorting analysis of an existing typical escort mechanism (I broaching start; II escorting stop; III broaching stop)

\section{Defects of existing escort mechanism}

The defects of the existing escort mechanism consist of three major aspects:

a) The escort mechanism moves up in opposite directions relative to the tool slide when escorting stops. The escort mechanism is provided enough motion space to moving up, so the tool slide must also be long enough to prevent collision between the escort mechanism and the workpiece. Consequently, the column is long.

b) There are two kinds of method, respectively mechanical method and electronic method, to prevent collision when escort stop. The mechanical method relies on a complex crash-avoidance mechanism, which prevents the escort mechanism from continuing to move down along with the tool slide. However, the crash-avoidance mechanism is complex and difficult to assemble and repair, while it occupies a big volume. The crucial point about the electronic method is that the electrical device sends a control signal, which triggers the oil cylinder actuator pushing the escort mechanism up along with the tool slide when escorting stop. However, the electrical device may be failure and produces error signal, which leads to the collision.

c) The escorting does not reach the designated position. The crash-avoidance mechanism occupies a space volume. In order to prevent escort mechanism and workpiece from collapsing into one another, electrical device usually sends signal to prematurely stop the escorting, which subsequently affects broaching stability and broaching precision.

\section{DESIGN OF A NEW-STYLE ESCORT MECHANISM}

\section{A. Design philosophy}

The design philosophy of the escort mechanism of vertical internal broaching machine lies in interpreting the synchronization differently. Conventional wisdom holds that the escort mechanism should be fixed on tool slide just like the pull block does, and all three are combined together to keep synchronization. However, a knock-on effect is produced on the escort mechanism that designed according to this thought. For example, both the escort mechanism and the pull block are installed on the tool slide. When they move down to he position that escorting stops, the tool slide and the pull block continue to move to finish broaching. Consequently, a crashavoidance mechanism has to be designed to move the escort mechanism up relative to the tool slide, which prevents escort mechanism and workpiece from colliding. New-style thought believes that the escort mechanism and slide board are independent, and can be movably connected and fused together to keep synchronization. It has advantage of avoiding colliding, and solving the problems existing in the traditional escort mechanism.

\section{B. Implement method}

The linear rails that used in escorting are installed on the column but on the tool slide, which means that the escort mechanism moves all alone down along linear rails installed on the column instead of being driven by tool slide. The downwards motion of the escort mechanism depends on both 
self-weight and small friction force in the opposite direction. When the escort mechanism moves down along the linear rails, there is friction force between them that is too small to be neglected. The escort mechanism moves down under the influence of gravity and touches the top of the tool slide, then, it moves on with the tool slide synchronously. The linear ball rail system not only guides accurately the downwards motion for the escort mechanism, but also guarantees the axiality between the broaching tool and escorting position. Because the escort mechanism moves down much more rapidly than the downwards broaching of the tool slide, consequently, the escort mechanism can lean tightly against the tool slide. Using this escort mechanism, the collision-avoidance system can be easily designed. Provided that the column is installed hanging block, the escort mechanism can be stopped at given position using the hanging blocks. The hanging blocks are symmetrically distributed at opposite ends of the column, and the tool slide can continue to move down with no effect. In addition, provided that the hanging blocks are installed at the low position of the column while the escorting stops, the vertical internal broaching machine gets the maximum stability of the broaching process.

\section{Detailed mechanical structure and escorting process}

Figure 3 shows the three-dimensional mechanical structure of the new-style escort mechanism of vertical internal broaching machine, as well as two demarcation positions of the escorting process, namely, broaching starts and escorting stops.

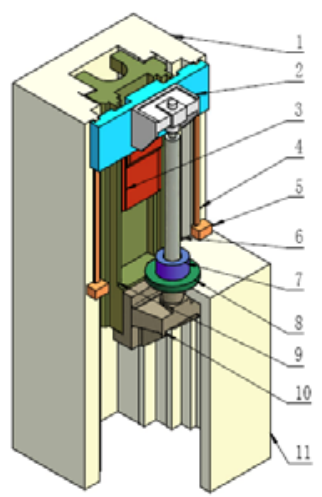

Stage I

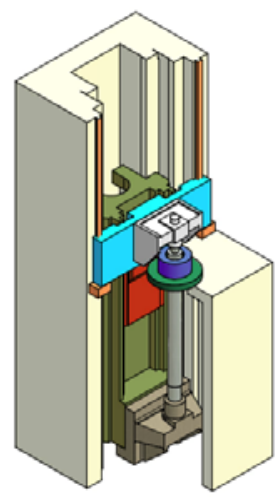

Stage II
Fig.3. Detailed mechanical structure of the new-style escort mechanism. (I broaching stats; II escort stops. 1-column 2- escort mechanism 3- carrying blocks 4- linear rails 5- hanging blocks 6- broaching tool 7- workpiece 8work table 9- tool slide 10- pull block 11- base table)

When broaching starts, the escort mechanism holds the tail of the broaching tool, and synchronously moves down with the tool slide along the column. The linear ball rail system installed in the column, meanwhile there are carrying blocks on the tool slide. The escort mechanism is lumped tightly with the carrying blocks as a whole during escorting, and moves down synchronously. When escorting stops, the escort mechanism is stopped by the hanging blocks that fixed in the column, and separates with the tool slide. The latter continues to move down until the broaching stop. The hanging blocks are installed at the low position of the column to guarantee the stability of the broaching process. The new-style escort mechanism of vertical internal broaching machine doubles the escorting distance, namely, two-thirds of the total length of the broaching tool, and realizes the true meaning full escort.

\section{PERFORMANCE OF THE NEW-STYLE ESCORT MECHANISM}

The new-style escort mechanism of vertical internal of broaching machine meets the three requirement of escorting that analyzed in foregoing paragraphs, namely, synchronization, safety and stability, which to some degree improve the traditional escort mechanism.

- When the escort mechanism moves along the column, the distance of the travel has nothing to do with the tool slide. That is, the latter needs not give any space for the escort mechanism to move up. As a consequence, the new-style escort mechanism uses shorter tool slide and the column. For example, a 40-ton broaching machine with stroke of two meters has a reduction of one meter in the length both of the tool slide and column, after using the new-style escort mechanism.

- $\quad$ Strong security. The pull block and the escort mechanism are combined together, as well as the way of hanging stop with mechanical carry during escorting, which help keep synchronization of broaching and escorting. When escorting stops, the pull block continues with the work, they do not influence each other. The escort mechanism will stop exactly at the position where the hanging blocks are located, the latter are fixed on the column. This eliminates the possibility of collision, and there are no complex collision avoidance system and electric control devices.

- In order to get the stability and accuracy of broaching over the process, the escorting stroke should be as long as possible. For this reason, the hanging blocks are installed at the lowest safe position where the escorting must stop. The distance between the escort mechanism and the workpiece using the new-style is fifteen times than that applying original system, and is reduced to within $20 \mathrm{~mm}$.

In addition, the new-style escort mechanism can be widely used in any vertical internal broaching machines with either single oil cylinder or double oil cylinders, provided that demanding full escorting. The vertical internal broaching machines with full escorting have combined the broaching mechanism, escort mechanism, tool carrying feed mechanism, and tool setting mechanism, into a single transmission mechanism. As a result, the number of parts is cut in the half. Morevoer, it has brought great convenience to whether manufacturing, assembling, or maintenance. In comparison with traditional broaching machine, the broaching machine applying the new-style escort mechanism improves the quality and at the same time reduces the cost, and is quite competitive in the market. 


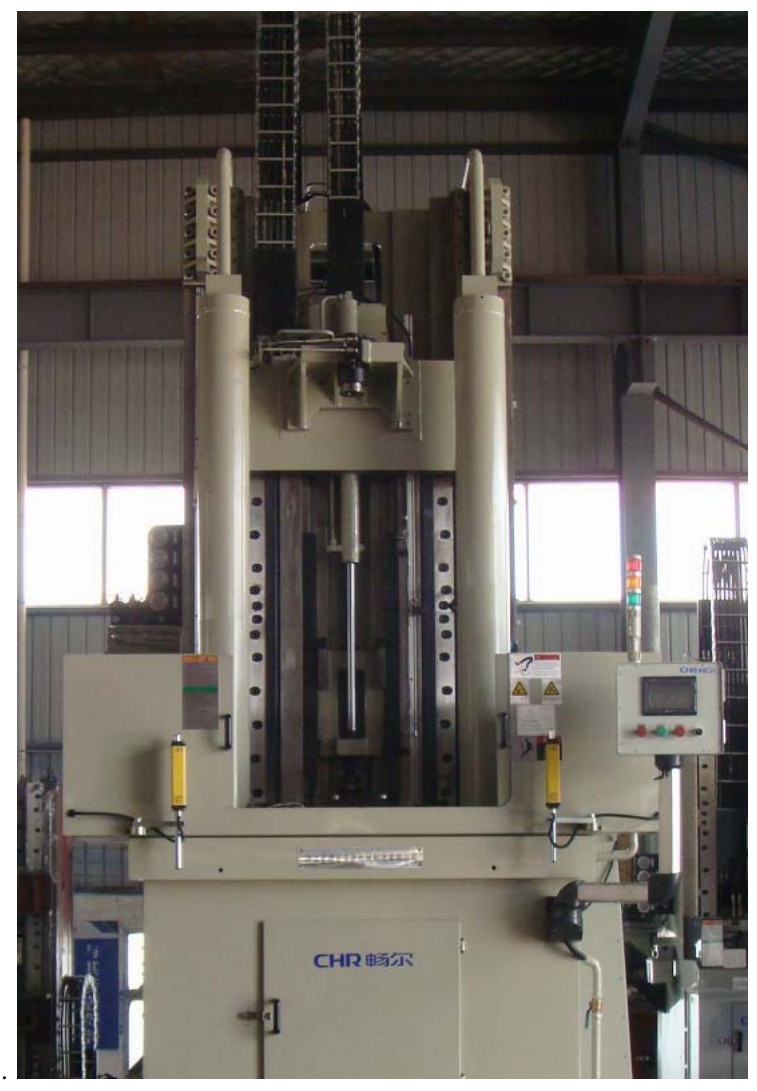

Fig.4. A application of the new-style escort mechanism

\section{CONCLUSION}

On the base of analysis of structure defects and escorting quality of traditional escort mechanism used in broaching machine, this paper presents a design result of a new-style escort mechanism of vertical internal broaching machine. The escort mechanism movably connects with the tool slide and is carried on the tool slide during escorting, and they synchronously move until escorting stop, by now they separate and the escort mechanism stops and hangs on the column. As a result, the new-style escort mechanism guarantees the synchronization, safety, and stability, and at the same time reduces the length of the tool slide and the column, further reduces costs.

The vertical internal broaching machines with either single oil cylinder or double oil cylinders, applying the new-style escort mechanism, have come into use so far. They were positioned as universal internal broaching machine, and these series will gradually replace the traditional internal broaching machine with full escorting. They will enhance the precision of domestic internal broaching machine, and expand the range of application.

\section{ACKNOWLEDGMENT}

The advice received from LU JINGJING has been inspirational and is gratefully appreciated

\section{REFERENCES}

[1] Sajeev, V., L. Vijayaraghavan, and U. Rao, Effect of tool-work deflections on the shape of a broached hole. International Journal of Mechanical Engineering Education, 2000. 28(1): p. 88-92.

[2] Kuljanic, E., Cutting force and surface roughness in broaching. Annals of the CIRP, 1975. 24(1): p. 77-82.

[3] Axinte, D.A., An experimental analysis of damped coupled vibrations in broaching. Journal of Machine Tools \& Manufacture, 2007. 47: p. 21822188.

[4] C, M., Broaching. 1960, London: The Machinery Publishing Co.

[5] Dragos, A.A., Approach into the use of probabilistic neural networks for automated classification of tool malfunctions in broaching. International Journal of Machine Tools \& Manufacture, 2006. 46: p. 1445-1448.

[6] Kokturk, U. and E. Budak. Optimization of broaching tool design. in Proceedings of the Intelligent Computation in Manufacturing Engineering. 2004. Sorrento.

[7] Shi, D. and N.N. Gindy, Development of an online machining process monitoring system: Application in hard turning. Sensors and Actuators A: Physical, 2007. 135(2): p. 405-414.

[8] Boud, F. and N. Gindy, Application of multi-sensor signals for monitoring tool/workpiece condition in broaching. International Journal of Computer Integrated Manufacturing, 2008. 21(6): p. 715-729. 